\title{
Aggregation Operators Review - Mathematical Properties and Behavioral Measures
}

\author{
David L. La Red Martínez \\ Department of Computer, National University of the Northeast, Corrientes, (3400), Argentine \\ E-mail:1rmdavid@exa.unne.edu.ar \\ Julio C. Acosta \\ Department of Computer, National University of the Northeast, Corrientes, (3400), Argentine \\ E-mail: julioaforever@ hotmail.com
}

\begin{abstract}
A problem that humans must face very often is that of having to add, melt or synthesize information, that is, combine together a series of data from various sources to reach a certain conclusion or make a certain decision. This involves the use of one or more aggregation operators capable to provide a collective preference relation. These operators must be chosen according to specific criteria taking into account the characteristic properties of each operator. Some conditions to be taken into account to identify them are the following: axiomatic strength, empirical setting, adaptability, numerical efficiency, compensation and compensation range, added behavior and scale level required of the membership functions. It is possible to establish a general list of possible mathematical properties whose verification might be desirable in certain cases: boundary conditions, continuity, not decreasing monotony, symmetry, idempotence, associativity, bisymmetry, selfdistributivity, compensation, homogeneity, translativity, stability, $\phi$-comparability, sensitivity and locally internal functions. For analyze the attitudinal character of the aggregation operator the following measures are studied: disjunction degree (orness), dispersion, balance and divergence. In this paper, a review of these issues is presented.
\end{abstract}

Index Terms-Aggregation, aggregation operators, behavioral measures of aggregation operators, intersection operators, OWA operators.

\section{INTRODUCTION}

A problem that humans must face is usually that of having to add, melt or synthesize information [1].

Aggregation involves the use of one or more operators capable of providing a collective preference relation [2].

The aggregation problem arises in virtually any discipline [3].

The different scenarios in which a system may need to add information are usually classified in two groups according to the nature of the problem [4]:

(a) Aggregation of information for decision-making.

(b) Adding information to the description or representation of objects.

An important aspect related with the study of aggregation operators is to analyze what properties must meet, or just which properties meet the proposed operators. There is not a single criterion for selecting aggregation operators and this has led some conditions to be taken into account to identify them [5] [6]:
(a) Axiomatic strength
(b) Empirical setting.
(c) Adaptability.
(d) Numerical efficiency.
(e) Compensation and compensation range.
(f) Added behavior.
(g) Scale level required of the membership functions.

An interesting question is to consider the attitudinal character of the aggregation operator. The following measures are defined:
(a) Disjunction degree (orness).
(b) Dispersion.
(c) Balance operator.
(d) Divergence.

The main aggregation operators have been divided according to their position on the minimum and maximum operators into four groups [7]:

A. Lower or equal to the minimum: a. T-norms.

B. Greater than or equal to the maximum: a. T-conorms.

C. Between the minimum and maximum:

a. Quasi-linear average:

1. Weighted average.

2. Quasi-arithmetic means.

3. Generalized means.

b. Weighted minimum and maximum introduced in the framework of possibility theory-.

c. Ordered weighted averaging:

1. ME-OWA. 


\section{S-OWA. \\ 3. Step-OWA. \\ 4. Window-OWA. \\ 5. Neat-OWA.}

d. Weighted ordered weighted averaging.

e. Fuzzy integrals.

D. Hybrids.

In this article, we provide a brief overview of the main aggregation operators, and it is also structured as follows: firstly, we make a brief review of the aggregation of information; secondly, it is presented the mathematical properties of aggregation; thirdly, we will present the main aggregation operators; and finally, it will finish with conclusions about them.

\section{REVIEW ON DATA AGGREGATION}

A problem that humans must face is usually that of having to add, melt or synthesize information, i.e. combined each other a series of data from various sources to reach a certain conclusion or make a certain decision [1].

Moreover, in everyday activity of organizations (including governments), decisions must be made on which depends the success of management. Generally, decision models are used including an aggregation phase and another of exploitation. Aggregation involves the use of one or more operators capable of providing a collective preference relation. Thus, aggregation of information in an efficient and flexible way has become the main task of the problems of access to information and other problems of multicriteria decision [2].

The aggregation problem arises in virtually any discipline, being the medicine, economics, statistical or control theory only a few significant examples. Therefore, search, study and formalization of methods and techniques for aggregating information constitute a research field of wide spectrum and great timeliness. In particular, the need for rigorous mechanisms for this task is particularly evident in the field of Governments or Administrations, since aggregation of information is essential in fields such as decision-making and acquisition of knowledge from large volumes of data, among others, resulting very useful the aggregations between the minimum and the maximum operator, through the means operators [3].

In any of these fields, the different scenarios in which a system may need to add information are usually classified in two groups according to the nature of the problem [4]:

- Aggregation of information for decision-making: encompasses all those situations where you have multiple views or different criteria and plans to make a decision as consistent as possible with the initial information.

- Adding information to the description or representation of objects: it is required that when you have multiple information about the same object, but complementary and from different sources - experts, sensors, etc. - and it is intended to build on them an overall description of the object.

On the other hand, it is easy to check that, in the vast majority of aggregation processes, preliminary information is often uncertain or imprecise. So, it is generally convenient to have a framework that allows us to represent and handle such vagueness.

Although there are several mathematical environments capable of working with imperfect knowledge (calculation of probabilities, possibility theory, evidence theory), perhaps the most important of them is the theory of fuzzy subsets or fuzzy logic.

Obviously, there is not a single criterion for selecting aggregation operators and this has led some conditions to be taken into account to identify them [5]:

- Axiomatic strength: On equal terms, an operator is better when less limited is by which axioms satisfies.

- Empirical setting: In addition to satisfying certain axioms or have certain formal qualities, operators should appropriately reflect reality.

- Adaptability: The operators must be adapted to the specific context in which they are, essentially by parameterization.

- Numerical efficiency: The computational effort calculation is especially important when you have to solve big problems. In fact, many times we must resort to heuristics techniques able to find quality solutions although they are not necessarily optimal [6].

- Compensation and compensation range: The greater the extent to which the membership functions of the aggregated fuzzy sets, the aggregation operator better represent the situations in which attributes are compensated each other.

- Added behavior: The degree of membership in a fuzzy set in the aggregate set very often depends on the number of sets combined.

- Scale level required of the membership functions of: Different operators may require different levels of scale membership information (nominal, interval, ratio or absolute) to be admissible. On equal terms, it is preferred an operator that requires the lowest level scale.

In turn, Dubois and Prade propose the following classification of aggregation operators based on their behavior [7]:

- Conjunctive or intolerant behavior: It is desired that all criteria are met to combine, and it is represented by any lower or equal to the minimum operator. The t-norms satisfy this requirement and therefore belong to this category.

- Disjunctive or tolerant behavior: Simply one of the criteria is met for an overall satisfaction; it is represented by any greater or equal to the maximum operator. In this case the t-conorms are suitable 
carriers.

- Behavior of commitment: In many cases, it is desired to obtain an intermediate result that does not reflect neither the total lack of compensation which is the conjunctive behavior nor the total compensation of disjunctive behavior. This behavior is present in all operators between the minimum and maximum.

The above classification has the disadvantage of being too general and is even, as the authors themselves acknowledge, incomplete, and there are many operators such as symmetric sums - presenting a hybrid behavior that does not correspond to any of the three categories previous [1].

Generically it can be said that the timely aggregation fuzzy subsets is translated into the application of a numeric operator of the form $F:[0,1]^{n} \rightarrow[0,1]$ which, when checking the boundary conditions $F(0, \ldots, 0)=0$ and $F(1, \ldots, 1)=1$ and it is monotonous and continuous, this is called aggregation operator.

Because of its many applications, the definition and study of such operators has proliferated, and now, there is a great deal of proposals in this regard [7] [8] [9].

\section{MAthematicAl Properties OF AgGREgAtion}

An important aspect related with the study of aggregation operators is to analyze what properties must meet, or just which properties meet the proposed operators. Regarding the first point, author usually considers that these operators must meet categorically no concrete property, although they cite some that considered natural, such as boundary conditions, monotony or the continuity of the operators [1].

However, it is possible to establish a general list of possible mathematical properties whose verification might be desirable in certain cases [10] [11] [7] [12] [13] [14] [15] [16] [17] [18] [19] [20].

The main properties are these:

- Boundary conditions: $F(0, \ldots, 0)=0, F(1, \ldots, 1)=1$.

- Continuity: $F$ is a continuous function on each of its variables. This property ensures that the existence of small variations in the data does not cause big jumps in the result.

- Not decreasing monotony (in each variable): For all $i \in\{1, \ldots, n\}, \quad$ if $x_{i}>x_{i}^{g}$, then $F\left(x_{1, \ldots,}, x_{i, \ldots,} x_{n}\right) \geq F\left(x_{1, \ldots,} x_{i, \ldots,}^{v} x_{n}\right)$. By this property, it is described that if the input data increases, the result of their combination cannot decrease.

- Symmetry (or commutative, neutrality, anonymity): Where $I=[0,1]$; for all $\left(x_{1}, \ldots, x_{n}\right) \in I^{n}$, $F\left(x_{1, \ldots,} x_{n}\right)=F\left(x_{\sigma(1), \ldots,} x_{\sigma(n)}\right) ; \sigma$ being any permutation of $\{1, \ldots, n\}$. This property states that the order of the input data should not affect the result obtained, all of which are treated in the same way.
- Idempotence (or unanimously, identity): Where $I=$ $[0,1]$; for all $x \in I, F(x, \ldots, x)=x$. The idempotence is a generalization of the boundary conditions anywhere in the universe. It states that if all input data are the same, the result of their combination should match with them.

- Associativity: $(n=2)$ For all $x_{1}, x_{2}, x_{3} \in I, F\left(x_{1}, F\left(x_{2}\right.\right.$, $\left.\left.x_{3}\right)\right)=F\left(F\left(x_{1}, x_{2}\right), x_{3}\right)$. This property allows extending immediate, consistent and unambiguous manner to operators defined on two variables at any number of arguments.

- Bisymmetry: $(n=2)$ For all $x_{1}, x_{2}, x_{3}, x_{4} \in I, F\left(F\left(x_{1}\right.\right.$, $\left.\left.x_{2}\right), \quad F\left(x_{3}, x_{4}\right)\right)=F\left(F\left(x_{1}, x_{3}\right), \quad F\left(x_{2}, x_{4}\right)\right)$. The bisymmetry property, weaker than the association, said that the result of combining in groups of two input data does not depend on the choice of these groups. All associative and commutative function is bisymmetric.

- Self-distributivity: $(n=2)$ For all $x_{1}, x_{2}, x_{3} \in I, F\left(x_{1}\right.$, $\left.F\left(x_{2}, x_{3}\right)\right)=F\left(F\left(x_{1}, x_{2}\right), F\left(x_{1}, x_{3}\right)\right)$ (by the left). $(n=2)$ For all $x_{1}, x_{2}, x_{3} \in I, F\left(F\left(x_{1}, x_{2}\right), x_{3}\right)=F\left(F\left(x_{1}, x_{3}\right)\right.$, $F\left(x_{2}, x_{3}\right)$ ) (by the right). Any idempotent and bisymmetric function is self-distributive. Any strictly increasing and self-distributive function is idempotent.

- Compensation (or property of Pareto, average or the media): For all $\left(x_{1}, \ldots, x_{n}\right) \in I^{n}, \min \left(x_{1}, \ldots, x_{n}\right) \leq$ $F\left(x_{1}, \ldots, x_{n}\right) \leq \max \left(x_{1}, \ldots, x_{n}\right)$. The property compensation ensures that the result will be a compromise value located between the minimum and the maximum of all the input data. If $F$ satisfies the property compensation, then $F$ is idempotent. If $F$ is no decreasing monotonic and idempotent, then $F$ satisfies the property compensation.

- Homogeneity: For all $\left(x_{1}, \ldots, x_{n}\right) \in I^{n}$ and for all $t \in \mathbb{R}$, $t>0, F\left(t x_{1}, \ldots, t x_{n}\right)=t F\left(x_{1}, \ldots, x_{n}\right)$.

- Translativity: For all $\left(x_{1}, \ldots, x_{n}\right) \in I^{n}$ and for all $t \in \mathbb{R}$, $F\left(x_{1}+t, \ldots, x_{n}+t\right)=F\left(x_{1}, \ldots, x_{n}\right)+t$.

- Stability (or invariance): For all $\left(x_{1}, \ldots, x_{n}\right) \in I^{n}$, $F\left(f\left(x_{1}\right), \ldots, f\left(x_{n}\right)\right)=f\left(F\left(x_{1}, \ldots, x_{n}\right)\right)$, where $f: \mathbb{R} \rightarrow \mathbb{R}$ continuous and increasing function. It is a generalization of the properties of homogeneity and translativity.

- $\phi$-Comparability: For all $\left(x_{1}, \ldots, x_{n}\right),\left(y_{1}, \ldots, y_{n}\right) \in I^{n}$, if $F\left(x_{1}, \ldots, x_{n}\right)<F\left(y_{1}, \ldots, y_{n}\right)$, then $F\left(\phi\left(x_{1}\right), \ldots, \phi\left(x_{n}\right)\right)$ $<F\left(\phi\left(y_{1}\right), \ldots, \phi\left(y_{n}\right)\right)$ where $\phi: \mathbb{R} \rightarrow \mathbb{R}$ an automorphism. The function $\phi$ is interpreted as a scale change. If $F$ is stable under a positive linear transformation, then $F$ is $\phi$-comparable. If $F$ is $\phi$ comparable and idempotent, then $F$ is stable under a positive linear transformation.

- Sensitivity: It is defined as a value that measures the behavior of an operator before the introduction of small changes in the input values. It is established two levels of sensitivity:

○ Extreme sensitivity (worst case): 


$$
\rho_{F}(\delta)=\bigvee_{\left|x_{i}-y_{i}\right| \leq \delta}|F(x)-F(y)|
$$

○ Medium sensitivity:

$$
S(F)=\int_{[0,1]^{n}} \sum_{i=1}^{n}\left(\frac{\partial F}{\partial x_{i}}\right)^{2}
$$

- The t-norm min and t-conorm max are those with less extreme sensitivity of all the t-norms and t-conorms.

○ The t-norm product $x y$ and t-conorm algebraic sum $x+y-x y$ are those with lower average sensitivity of all the t-norms and t-conorms.

- The (extreme or average) sensitivity of a t-norm coincides with sensitivity (extreme or average) of its dual $\mathrm{t}$-conorm respect to the standard negation.

- Locally internal functions: For all $\left(x_{1}, \ldots, x_{n}\right) \in I^{n}$, $F\left(x_{1}, \ldots, x_{n}\right) \in\left\{x_{1}, \ldots, x_{n}\right\}$. A trivial example of operators who verified it is order statistics. Note that some aggregation operators - as weighted averages or OWA - turn out to be convex linear combinations of local internal functions.

\section{BRIEF OVERVIEW OF THE MAIN AGGREGATION OPERATORS}

Including operators have been divided according to their position on the minimum and maximum operators into four groups [7]:

A. Lower or equal to the minimum: Those who demand that all aggregates criteria are met simultaneously, and therefore the result of the aggregation will be bounded above by the lower of the different grades of aggregate satisfaction. This class, whose components are commonly referred to intercept operators, includes known triangular norms.

B. Greater than or equal to the maximum: Those that generate a result that is bounded below by the larger of the added items. They are called union operators and its greatest exponent is the family of triangular conforms.

C. Between the minimum and maximum: Those who, unlike the previous two extreme cases described an attitude of compensation or averaging values returning a value between both extremes, and that could be termed average operators.

D. Hybrids: Are all those with a mixed attitude and therefore do not belong to any of the above three groups.

\section{A. Operators of intersection $(F \leq \mathrm{min})$}

In this group are especially distinguished triangular norms (t-norms) [1].

\section{a. T-norms}

The maximum exponent of intersection operators constitute the triangular norms or t-norms [10] [21] [22] [23] [24] [12] [13] [25] [26] [27] [28] [29] [30] [31] [32] [33] [34] [35].

Definition: Where $I=[0,1]$, a triangular norm or $\mathrm{t}$ norm is a function $T: I \times I \rightarrow I$ that satisfies the following properties for any $x, y, z, t \in I$ :

- $T(x, y)=T(y, x)$ (commutativity).

- $T(x, T(y, z))=T(T(x, y), z)$ (associativity).

- If $x \leq z$ and $y \leq t, T(x, y) \leq T(z, t)$ (monotony).

- $T(x, 1)=x$ (neutral element 1$)$.

\section{B. Union operators $(F \geq \max )$}

In this group are particularly distinguished triangular conorms (t-conorms) [1].

\section{a. T-conorms}

Definition: Where $I=[0,1]$; a triangular conorm or tconorm is a function $S: I \times I \rightarrow I$ that satisfies the following properties for any $x, y, z, t \in I$ :

- $S(x, y)=S(y, x)$ (commutativity).

- $S(x, S(y, z))=S(S(x, y), z)$ (associativity).

- If $x \leq z$ and $y \leq t, S(x, y) \leq S(z, t)$ (monotony).

- $S(x, 0)=x$ (neutral element 0$)$.

The t-conorms are obtained by duality from the $\mathrm{t}$ norms: a function $S: I \times I \rightarrow I$ is a t-conorm if $T(x, y)=1$ $S(1-x, 1-y)$ is a t-norm.

\section{Average operators $(\min \leq F \leq \max$ )}

These operators ensure obtaining an intermediate result between the minimum and maximum. They have the property to be idempotent. The most important families of operators of this type are [1]:
a. Quasi-linear average.
b. Weighted minimum and maximum -introduced in the framework of possibility theory-.
c. Ordered weighted averaging.
d. Weighted ordered weighted averaging.
e. Fuzzy integrals.

\section{a. Quasi-linear average}

These operators have also been extensively studied [10] [7] [36] [8] [37] [38] [39] [40] [41].

Definition: A quasi-linear medium is a function $M_{f, w}: I^{n}$ $\rightarrow I$ defined for all $\left(x_{1}, \ldots, x_{n}\right)$ of $I^{n}$, by: 


$$
M_{f_{u} w}\left(x_{1, \ldots,} x_{n}\right)=f^{-1}\left(\sum_{i=1}^{n} w_{i} f\left(x_{i}\right)\right)
$$

where $f: I \rightarrow \mathbb{R}$ a continuous and strictly monotone function called generating function of the mean and $w^{t}=$ $\left(w_{1}, \ldots, w_{n}\right)$ a vector of weights such that $w_{i} \in I$ and verifying that $\sum_{i=1}^{n} w_{i}=1$.

The main sub-classes of this family of operators are indicated:

1. Weighted average.

2. Quasi-arithmetic means.

3. Generalized means.

\section{Weighted average}

The weighted averages are a particular case of quasilinear averages constructed taking as generating function the identity function, $f(x)=x$ :

Definition: A weighted average is a function $M_{w}: I^{n} \rightarrow I$ defined for all $\left(x_{1}, \ldots, x_{n}\right)$ of $I^{n}$ by:

$$
M_{w}\left(x_{1, \ldots,} x_{n}\right)=\left(\sum_{i=1}^{n} w_{i} x_{i}\right)
$$

where $w^{t}=\left(w_{1}, \ldots, w_{n}\right)$ a vector of weights such that $w_{i} \epsilon$ $I$ and verifying that $\sum_{i=1}^{n} w_{i}=1$.

\section{Quasi-arithmetic means}

The quasi-arithmetic means are a particular case of quasi-linear means in which the weight vector is such that $w_{i}=1 / n$ for all $i \in\{1, \ldots, n\}$ :

Definition: A quasi-arithmetic mean is a function $M_{f}: I^{n}$ $\rightarrow I$ defined for all $\left(x_{1}, \ldots, x_{n}\right)$ of $I^{n}$ by:

$$
M_{f}\left(x_{1, \ldots,} x_{n}\right)=f^{-1}\left(\frac{1}{n} \sum_{i=1}^{n} f\left(x_{i}\right)\right)
$$

where $f: I \rightarrow \mathbb{R}$ a continuous and strictly monotone function called generating function of the mean.

\section{Generalized Means}

A particular and very common case of quasi-linear means are generalized means, obtained when the generating function is $f(x)=x^{\alpha}$ with $\alpha \in \mathbb{R}^{*}$ :

Definition: A generalized mean is a function $M_{\alpha, w}: I^{n} \rightarrow I$ defined for all $\left(x_{1}, \ldots, x_{n}\right)$ of $I^{n}$ by:

$$
M_{\alpha_{W} W}\left(x_{1, \ldots,} x_{n}\right)=\left(\sum_{i=1}^{n} w_{i} x_{i}^{\alpha}\right)^{\frac{1}{\alpha}}
$$

being $\alpha$ a parameter belonging to $\mathbb{R} *$ and $w^{t}=\left(w_{1}, \ldots, w_{n}\right)$ a weights vector such that $w_{i} \in I$ and verifying that $\sum_{i=1}^{n} w_{i}=1$

This last family include the following operators, obtained by taking the weights vector $w_{i}=1 / n$ for all $i \epsilon$ $\{1, \ldots, n\}$ :

- Minimum: $M_{-m}\left(x_{1}, \ldots, x_{n}\right)=\min \left(x_{1}, \ldots, x_{n}\right)$.

- Harmonic mean: $M_{-1}\left(x_{1, \ldots,} x_{n}\right)=\frac{1}{\frac{1}{x_{1}}+\cdots+\frac{1}{x_{n}}}$.

- Geometric mean: $\mathrm{M}_{0}\left(\mathrm{x}_{1}, \ldots, \mathrm{x}_{\mathrm{n}}\right)=\left(\mathrm{x}_{1} \mathrm{x}_{2} \ldots\right.$ $\left.\mathrm{x}_{\mathrm{n}}\right)^{1 / \mathrm{n}}$.

- Arithmetic mean: $M_{1}\left(x_{1, \ldots,} x_{n}\right)=\left(\frac{1}{n} \sum_{i=1}^{n}\left(x_{i}\right)\right)$.

- Maximum: $M_{+=0}\left(x_{1}, \ldots, x_{n}\right)=\max \left(x_{1}, \ldots, x_{n}\right)$.

\section{b. Weighted minimum and maximum}

The weighted minimum and maximum were developed as a generalization of the min and max operators [11] [7] [42]:

Definition: It is called weighted minimum and weighted maximum, respectively, to the functions of $w$-min, $w$-max $I^{n} \rightarrow I$ defined for all $\left(x_{1}, \ldots, x_{n}\right)$ of $I^{n}$ by:

$w-\min \left(x_{1, \ldots}, x_{n}\right)=\min \left(\max \left(x_{i}, 1-w_{i}\right)\right)(i=1, \ldots, n)$

and

$w-\max \left(x_{1, \ldots,} x_{n}\right)=\max \left(\min \left(x_{i}, w_{i}\right)\right)\left(i=1_{s, n} n\right)$

being $w^{t}=\left(w_{1}, \ldots, w_{n}\right)$ a normalized weights vector such that $\max \left(w_{i}\right)=1(i=1, \ldots, n)$.

The first operator describes a measure of need, while the second describes a measure of possibility. When all criteria to aggregate are equally important $\left(w_{i}=1\right.$ for all $i$ ), the minimum and maximum operators are obtained, respectively.

\section{c. Ordered Weighted Averaging (OWA)}

The Ordered Weighted Averaging (OWA) was introduced by Yager in 1988 as a new compensation operator. They allow the introduction of weights, and therefore they are similar to the weighted mean. The fundamental difference between the weighted mean and the new operator is that, in the latter case, the weights do not affect a specific criteria, weights affect the position of each criterion when the criteria are sorted: each weight $w_{i}$ is associated with the $i$-th largest element, regardless of whether [43] [44] [6] [45] [46] [47] [48] [49] [50] [51] [52] [53] [54] [55] [56] [57] [58] [59] [60] [61].

Definition: An OWA operator is defined as $F: R^{n} \rightarrow R$, it is associated with a vector of $n$ elements $W=\left[w_{1}, w_{2}, \ldots, w_{n}\right]^{T}$ such that $w_{i} \in[0,1]$ and $\sum_{i=1}^{n} w_{i}=1$

Further $F\left(a_{1}, a_{2}, \ldots, a_{n}\right)=\sum_{j=1}^{n} w_{j} \cdot b_{j}$ where $b_{j}$ is the $j$ 
largest of the $a_{i}$

A fundamental aspect of the OWA operators is the step of reorder. An aggregate $x_{i}$ is not associated with a particular weight $w_{j}$, a weight is associated with a particular $j$ position of the ordered arguments. This arrangement introduces nonlinearity in the aggregation process [62].

OWA operators have been applied in different areas, such as multicriteria decision-making [43] [63], expert systems [64] and fuzzy group decision making process [65] [55].

Noted that different OWA operators are distinguished by their weight function or weight. In [43] listed three important special cases of aggregations OWA:

1. $F^{*}$. In this case $W=W^{*}=[1,0, \ldots, 0]^{T}$.

2. $F_{*}$. In this case $W=W_{*}=[0,0, \ldots, 1]^{T}$.

3. $F_{\text {ave. }}$ In this case $W=W_{\text {ave }}=\left[\frac{1}{n}, \frac{1}{n}, \ldots, \frac{1}{n}\right]^{T}$.

Different approaches have been suggested for the determination of the weightings used in the OWA operator, for example, maximum entropy, method of learning, fuzzy quantifiers, minimum variability, etc. [65].

One of them allows to obtain weights according to linguistic quantifiers. In this case the quantifiers are defined as a function $Q:[0,1] \rightarrow[0,1]$ where $Q(0)=0$, $Q(1)=1$ and $Q(x) \geq Q(y)$ to $x>y$.

Zadeh [66] define the function $Q$ as follows:

$$
Q(x)=\left\{\begin{array}{lll}
0 & \text { if } & x<a \\
\frac{x-a}{b-a} & \text { if } & a \leq x \leq b \\
1 & \text { if } & b<x
\end{array}\right.
$$

with $a, b, x \in[0,1]$.

For a given value $x \in[0,1], Q(x)$ is the degree to which $x$ satisfies the fuzzy concept represented by the quantifier. Based on the $Q$ function, the OWA vector is determined from $Q$ as follows:

$$
w_{i}=Q\left(\frac{i}{n}\right)-Q\left(\frac{i-1}{n}\right)
$$

These weights have the function to increase or diminish the importance of the different components of the aggregation according to the semantics associated with $Q$, i.e., the quantifier determines the strategy of construction of weighting vector.

OWA operators could be considered as a particular case of a larger family of operators, which could be called quasi-linear ordered mean, and that would be given by the following definition [1]:

Definition: A quasi-linear ordered mean is a function $O_{f, w}: R^{n} \rightarrow R$ defined for all $\left(x_{1}, \ldots, x_{n}\right)$ of $R^{n}$, by:

$$
o_{f_{u}}\left(x_{1, \ldots,} x_{n}\right)=f^{-1}\left(\sum_{i=1}^{n} w_{i} f\left(x_{\sigma(\mathbb{i})}\right)\right)
$$

where $f: R \rightarrow R$ continuous and strictly monotonic function called generating function of the mean, $w^{t}=$ $\left(w_{1}, \ldots, w_{n}\right)$ a vector of weights such that $w_{i} \in R$ and verifying $\sum_{i=1}^{n} w_{i}=1$, and where $\{\sigma(1), \ldots, \sigma(n)\}$ is a permutation of $\{1, \ldots, n\}$ such that $x_{\sigma(\mathrm{i}-1)} \geq x_{\sigma(\mathrm{i})}$ for all $i=2, \ldots, n$.

An OWA is a compensation (and therefore idempotent) operator, monotone, none decreasing in each variable, commutative and homogeneous.

The arithmetic mean is an OWA particular case, obtained by taking all weights equal to $1 / n$. The same, it goes for the so-called order statistics, obtained to the vector of weights formed entirely by zeros except for one in the right position, and that turn includes maximum and minimum operators.

Definition: The $k$-th order statistical $(k \in\{1, \ldots, n\})$ is a function $x^{(k)}: R^{n} \rightarrow R$ defined, for all $\left(x_{1}, \ldots, x_{n}\right)$ of $R^{n}$, by:

$$
x^{(k)}\left(x_{1, \ldots,} x_{n}\right)=y_{k}
$$

where $y_{k}$ is the $k$-th smallest element of $\left(x_{1}, \ldots, x_{n}\right)$.

Some of the most common OWA operators are as follows [67]:

1. ME-OWA.

2. S-OWA.

3. Step-OWA.

4. Window-OWA.

5. Neat-OWA.

\section{1. $M E-O W A$}

The first family of parameterized OWA operators is defined by O'Hagan [64]. This family of operators is called ME-OWA, the ME acronym referring to maximum entropy.

The procedure developed for the calculation of the weights is as follows: Firstly, you select a desired value of orness (taking an optimistic value) $\alpha$; then those weights that allow you to get the desired $\alpha$ value are determined with maximum dispersion (entropy). In particular, the following programming problem is resolved:

Maximize

$$
\left(-\sum_{i}^{n}\left(w_{i} \ln w_{i}\right)\right)
$$

Fulfilling known restrictions

$$
\sum_{i=1}^{n} w_{i}=1
$$




$$
w_{i} \in[0,1]
$$

To which it is added

$$
\alpha=\frac{1}{n-1} \sum_{i}^{n}(n-i) \cdot w_{i}
$$

It can be observed how, through a single $\alpha$ parameter, the total of the weights of the desired system are obtained, according to the philosophy of the maximum entropy technique. An extension of ME-OWA operators is used as a measure of entropy $1-\operatorname{Max}_{i}\left[w_{i}\right]$, which is to be as objective function to minimize the $\operatorname{Max}_{i}\left[w_{i}\right]$.

\section{S-OWA}

Another family of OWA operators are the S-OWA. These operators are classified into two subfamilies depending on whether they are, or type or and type.

The S-OWA operators of type or are denoted as FSO, the weights are defined as follows:

$$
w_{i}= \begin{cases}\frac{1}{n}(1-\alpha)+\alpha & i=1 \\ \frac{1}{n}(1-\alpha) & i=2, \ldots, n\end{cases}
$$

Using this definition, you get a form of aggregation of interest:

$$
F_{S O}\left(a_{1}, \ldots, a_{n}\right)=\alpha \operatorname{Max}_{i}\left(a_{i}\right)+\frac{1}{n}(1-\alpha) \sum_{i} a_{i}
$$

This allows you to generate a weighted average between the maximum and the average of the values aggregated.

If $\alpha=0$ is obtained $\frac{1}{n} \sum a_{i}$ and if $\alpha=1 \operatorname{Max}\left(a_{i}\right)$.

Namely:

$$
F_{S O}=\alpha F^{*}+(1-\sigma) F_{A} .
$$

For this form of aggregate, orness measure is calculated as follows:

$$
\operatorname{orness}\left(F_{S O}\right)=\frac{1}{2}(\alpha+1)
$$

It is shown how to $\alpha \in[0,1]$, the value of orness is placed in $[0.5,1]$. For this reason, FSO operator can be seen as a measure of or type. Furthermore, with increasing orness, increases the value of $\alpha$. Particularly when $\alpha=1$, get an orness(FSO) $=1$ and when $\alpha=0$, orness $(\mathrm{FSO})=0.5$, so in this case we will be using a simple average.

The second class of S-OWA operators, denoted as FSA, are the cataloged within the and type, and are defined as follows:

$$
\begin{gathered}
w_{i}=\frac{1}{n}(1-\beta), \quad i \neq n \\
w_{n}=\frac{1}{n}(1-\beta)+\beta
\end{gathered}
$$

where $\beta \in[0,1]$. Using these weights, we get:

$$
F_{S A}\left(a_{1}, \ldots, a_{n}\right)=\beta \operatorname{Min}_{i}\left(a_{i}\right)+\frac{1}{n}(1-\beta) \sum_{i} a_{i}
$$

In this case you get a weighted average between the average and minimum values of the set to aggregate. It is clear that:

$$
F_{S A}=\beta F_{*}+(1-\beta) F_{A}
$$

Being its orness measure:

$$
\operatorname{orness}\left(F_{S A}\right)=\frac{1}{2}(1-\beta) \text {. }
$$

You will always have values between 0.5 and 0 . Thus, if it is calculated the andness, gets a value which will be located between 1 and 0.5. As in the previous case, if $\beta=0 \mathrm{FAV}$ is obtained, meaning that the arithmetic mean or FAV is an operator positioned between and and or.

These operators have very useful properties. First, given a value of orness, $\lambda$, it is very easy to generate the weights associated with the aggregation expressed by this value.

If $\lambda \geq 0.5$ used one type or S-OWA with $\alpha=2 \lambda-1$.

If $\lambda<0.5$ used one type and S-OWA with $\beta=1-2 \lambda$.

Once obtained the weights, aggregation calculation is very simple, only the sum of the elements is necessary more the Max or Min of aggregation.

In Ref. [68] this type of operators is used to generate a new class of flexible logic controllers with fuzzy logic.

This operator has the advantage of permitting to combine the two mentioned families of $\alpha, \beta$ operators, producing a general S-OWA operator. It is considered $\alpha, \beta \in[0,1]$ with $\alpha+\beta \leq 1$, is defined:

$$
\begin{gathered}
w_{1}=\frac{1}{n}(1-(\alpha+\beta))+\alpha \\
w_{i}=\frac{1}{n}(1-(\alpha+\beta)), \mathrm{i}=2,3, \ldots, \mathrm{n}-1 \\
w_{n}=\frac{1}{n}(1-(\alpha+\beta))+\beta
\end{gathered}
$$


This generalization is denoted as $F_{s}$ being the general formula:

$$
F_{s}\left(a_{1}, \ldots, a_{n}\right)=\alpha \operatorname{Max}_{i}\left[a_{i}\right]+\beta \operatorname{Min}_{i}\left[a_{i}\right]+(1-(\alpha+\beta)) \sum_{i} a_{i}
$$

\section{Step-OWA}

The Step-OWA operators or type step operators (Yager, 1993) [69], denote as $F_{\text {step }}(k)$ and define their weights as follows:

$w_{k}=1$

$\mathrm{W}_{\mathrm{i}}=0, \mathrm{i} \neq \mathrm{k}$

As shown, with step-OWA operators, one nonzero weight, which corresponds exactly to the weight $k$ is obtained. If $k=1$ we obtain the operator $F^{*}$, while when $k=n$ we obtain the operator $F_{*}$.

Is easy to see that $F_{\text {step }(k)}=\left(a_{1}, \ldots, a_{n}\right)=b_{k}$ where $b_{k}$ corresponds to the major $k$-th element of all elements, and this is the result of the aggregation.

The dispersion associated with this operator can be calculated as:

$$
\operatorname{Disp}\left(F_{s t e p(k)}\right)=-\sum w_{i} \ln w_{i}=0
$$

So it is regarded as an aggregation of minimum entropy.

The measure of orness associated with this operator is calculated as:

$$
\operatorname{orness}\left(F_{\text {step }(k)}\right)=\frac{1}{n-1} \sum_{i=1}^{n}(n-i) \cdot w_{i}=\frac{n-k}{n-1}
$$

For these quantifiers $w_{k}=1$ if $\frac{k-1}{n}<\gamma \leq \frac{k}{n}$. Always $\gamma>0$.

Semantically this quantifier is interpreted as at least $\gamma$ percent. If $n$ is considered fixed, then it is interpreted as at least $n \gamma$.

\section{Window-OWA}

The window type operators are characterized by using two parameters, $k$ and $m$, in order to determine the aggregation weights. These operators will denote as $F_{w}$ and defined as follows:

$$
w_{i}=\left\{\begin{array}{rrr}
0 & \text { if } & i<k \\
\frac{1}{m} & \text { if } & k \leq i \leq k+m \\
0 & \text { if } & i \geq k+m
\end{array}\right.
$$

Being $k$ and $m$ positive integers such that $k+n \leq n+1$, where $n$ is the cardinality of the OWA aggregation. It is easy to check that the window type operators have a total of $m$ nonzero weights and all with the identical value $\frac{1}{m}$, being $k$ the position where the nonzero vector starts.

A typical vector for this case is, for

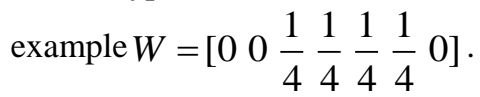

Using these weights general formula is obtained for the operator:

$$
F_{w}\left(a_{1}, \ldots, a_{n}\right)=\frac{1}{m} \sum_{j=k}^{k+m-1} b_{j}
$$

where $b_{j}$ is the $j$-th largest value of $a_{i}$.

As you seen, this operator provides a window to the collection of sorted elements starting at position $k$, within the window is calculated an average of elements of the aggregation.

The entropy or dispersion associated with this type of aggregation is easily calculable through this expression:

$$
\operatorname{Disp}\left(F_{w}\right)=-\sum_{i=1}^{n} w_{i} \ln w_{i}=-\sum_{i=k}^{k+m-1} \frac{1}{m} \ln \frac{1}{m}=-\ln \frac{1}{m}=\ln m
$$

It is interesting to see how the dispersion is always relative to the number of elements that are aggregated, if the number of elements increases, increases the dispersion.

The degree of orness associated with this operator is calculated with the following expression:

$$
\begin{gathered}
\operatorname{orness}\left(F_{w}\right)=\frac{1}{n-1} \sum_{i=1}^{n}(n-i) w_{i} \\
\operatorname{orness}\left(F_{w}\right)=\frac{1}{n-1} \sum_{i=k}^{k+m-1}(n-i) w_{i}=\frac{1}{n-1} \frac{1}{m} \sum_{i=k}^{k+m-1}(n-i)=\frac{1}{n-1}\left(n-k-\frac{1}{2}(m-1)\right)
\end{gathered}
$$

To increase $k$ or $m$ decreases the $\operatorname{orness}\left(F_{w}\right)$. You can check in the case that $m$ is 1 to get a quantifier of the step type.

\section{Neat-OWA}

Other families of OWA operators of greater importance are the so-called neat-OWA, characterized, in this case, because the weights depend on the values to aggregate.

In defining the OWA operators indicated that $f\left(a_{1}, a_{2}, \ldots, a_{n}\right)=\sum_{j=1}^{n} w_{j} \cdot b_{j}$, where $b_{j}$ is the $j$-th largest value of $a_{n}$, and where the weights are restricted to satisfy (1) $w_{i} \in[0,1]$ and (2) $\sum_{i=1}^{n} w_{i}=1$.

In all definitions above, it is assumed that weights are constant fixed values. However, for this family of operators the weights will be calculated on the basis of the elements that are aggregated, or more exactly from the values to aggregate ordered, the $b_{j}$, conditions being maintained (1) y (2). In this case the weights are: 
$w_{i}=f_{i}\left(b_{1}, \ldots, b_{n}\right)$, and the operator is defined as follows:

$$
F\left(a_{1}, \ldots a_{n}\right)=\sum_{i} f_{i}\left(b_{1}, \ldots, b_{n}\right) b_{i}
$$

For this family, where the weights depend on the aggregation, the satisfaction of all the properties of the OWA operators is not required.

Any aggregation of elements must be between the values produced by the functions $F^{*}$ y $F_{*}$.

The operator is idempotent $F(a, \ldots, a)=a$.

The operator is commutative, that is, the order of the $a_{i}$ elements is not relevant.

A property that is not necessarily satisfied for this family of operators is the monotony.

We consider $A=\left(a_{1}, \ldots, a_{n}\right)$ and $C=\left(c_{1}, \ldots, c_{n}\right)$, two sets to be aggregated such that $a_{i} \geq c_{i}$ for all $i$. If the weights are kept constant then:

$$
F(A) \geq F(C)
$$

As you can see, as the weights depend on the elements to aggregate, if you change the values to aggregate, $w_{i}$ also may change, so it cannot be assured that this property for all cases is met.

Moreover, to say that an aggregation operator is neat, it is necessary that the final value of aggregation should be independent of the order of the values.

Consider $A=\left(a_{1}, \ldots, a_{n}\right)$ entries to add, $B=\left(b_{1}, \ldots, b_{n}\right) \quad$ ordered entries and $C=\left(c_{1}, \ldots, c_{n}\right)=\operatorname{Perm}\left(a_{1}, \ldots, a_{n}\right)$ a permutation of the entries. It is formally defined as a neat-OWA operator if:

$$
F\left(a_{1}, a_{2}, \ldots, a_{n}\right)=\sum_{i=1}^{n} w_{i} \cdot b_{i}
$$

It produces the same result for any assignment $C=B$.

A typical example of neat-OWA operator is when $w_{i}=\frac{1}{n}$. So:

$$
F\left(a_{1}, a_{2}, \ldots, a_{n}\right)=\frac{1}{n} \sum_{i=1}^{n} a_{i}
$$

In this case, as the weights are fixed, this is the only expression of calculation for the operator. On the other hand, as the weights depend on the values to aggregate, you can define different types of neat operators within the same family.

One of the characteristics of the neat OWA operators is that it is not necessary to order the values to aggregate to your process. This implies that the formulation of a neat operator can be defined using directly the arguments instead of the ordered items.
First families of operators whose weights depend on the aggregation are known as BADD-OWA [68].

In this case, the operator defines their weights as:

$$
w_{i}=\frac{b_{i}^{\alpha}}{\sum_{i} b_{i}^{\alpha}}, \alpha \geq 0
$$

You can check that the conditions are satisfied:

1. $w_{i} \in[0,1]$

2. $\sum_{i=1}^{n} w_{i}=1$.

So the weights function can be accepted as valid. For this operator function would be as follows:

$$
F\left(a_{1}, a_{2}, \ldots, a_{n}\right)=\frac{\sum_{i} b_{i}^{\alpha+1}}{\sum_{i} b_{i}^{\alpha}}
$$

Where can you easily verify that the operator is neat category and does not need the ordering process of the arguments to aggregate:

$$
F\left(a_{1}, a_{2}, \ldots, a_{n}\right)=\frac{\sum_{i} a_{i}^{\alpha+1}}{\sum_{i} a_{i}^{\alpha}}, \quad \alpha \geq 0
$$

It looks how when $\alpha=0$ is obtained:

$$
F\left(a_{1}, a_{2}, \ldots, a_{n}\right)=\frac{1}{n} \sum_{i} a_{i}
$$

which corresponds to the simple average or arithmetic mean $F_{\text {ave }}$.

When $\alpha=1$, is obtained:

$$
F\left(a_{1}, a_{2}, \ldots, a_{n}\right)=\frac{\sum_{i} a_{i}^{2}}{\sum_{i} a_{i}} .
$$

When $\alpha \rightarrow \infty$ is obtained:

$$
F\left(a_{1}, a_{2}, \ldots, a_{n}\right)=\operatorname{Max}\left[a_{i}\right]=F^{*}\left(a_{1}, a_{2}, \ldots, a_{n}\right)
$$

In Ref. [68] it is shown how these operators are not monotonic with respect to the arguments. To verify the not monotony, we will consider that $n=2$ and $\alpha=1$. In this case:

$$
F\left(a_{1}, a_{2}\right)=\frac{a_{1}^{2}+a_{2}^{2}}{a_{1}+a_{2}}
$$


If we assign values $a_{1}=1$ and $a_{2}=0.2$, then the aggregate function applies:

$$
F(1,0.2)=\frac{1+0.04}{1.2}=0.866
$$

If we assign values $a_{1}=1$ and $a_{2}=0.3$, in this case the aggregated function worth:

$$
F(1,0.3)=\frac{1+0.09}{1.3}=0.838
$$

Although the value corresponding to the second term has been increased by one tenth of the unit.

Other many traditional operators can be classified as neat OWA operators, since they meet the required properties to be grouped as members of this class, examples of these operators are the arithmetic mean or the harmonic mean.

\section{d. Weighted Ordered Weighted Averaging (WOWA)}

In 1997, Torra suggests a new operator for the combination of information, called Weighted Ordered Weighted Averaging (WOWA), built as a mixture of two operators: classical weighted averages and ordered weighted averages (OWA) of Yager [32].

Its definition is as follows:

Definition: A WOWA is a function $O_{w, p}: R^{n} \rightarrow R$ defined, for all $\left(x_{1}, \ldots, x_{n}\right)$ of $R^{n}$ by:

$$
o_{w, p}\left(x_{1}, \ldots, x_{n}\right)=\sum_{i=1}^{n} \lambda_{i} x_{\sigma(\hat{i})}
$$

where $w^{t}=\left(w_{1}, \ldots, w_{n}\right)$ and $p^{t}=\left(p_{1}, \ldots, p_{n}\right)$ are vectors such that $w_{i}, p_{i} \in R$ verifying $\sum_{i=1}^{n} w_{i}=\sum_{i=1}^{n} p_{i}=1$, $\{\sigma(1), \ldots, \sigma(n)\}$ is a permutation of $\{1, \ldots, n\}$ such that $x_{\sigma(i-1)} \geq x_{\sigma(\mathbb{i})}$ for all $i=2, \ldots, n$ and weights $\lambda_{i}$ are defined as:

$$
\lambda_{i}=W^{*}\left(\sum_{j \leq i} p_{\sigma(j)}\right)-W^{*}\left(\sum_{j \in i} p_{\sigma(j)}\right)
$$

being $\mathrm{W}^{*}: R \rightarrow R$ an increasing monotonic function that interpolates the points $\left(\frac{i}{n}, \sum_{j \leq i} w_{i}\right)$ along with point $(0,0)$.

\section{e. Fuzzy integrals}

The basic definitions are as follows [70] [71] [72] [73]:

Definition: $\mathrm{Be} X=\left\{x_{1}, \ldots, x_{n}\right\}$ a set of criteria and $P(X)$ all the parts of $X$. A fuzzy measure is a function $\mu: P(X) \rightarrow I$ which verifies the following axioms: a) $\mu(\phi)=0, \mu(X)=1$; b) si $A \subseteq B$ then $\mu(A) \leq \mu(B)$ for any $A, B \in P(X)$.
Definition: Be $\mu$ a fuzzy measure defined on a set of criteria $X=\left\{x_{1}, \ldots, x_{n}\right\}$. The Sugeno discrete integral of $n$ values $a_{1}, \ldots, a_{n}$ of $[0,1]$ is defined in the following way:

$$
s_{\mu}\left(a_{1, \ldots,}, a_{n}\right)=\bigvee_{i=1}^{n}\left(a_{(\mathbb{i})} / \mu\left(A_{(\mathbb{i})}\right)\right)
$$

being $a_{(i)}$, with $i \in\{1, \ldots, n\}$, a permutation of $a_{i}$ such that $a_{(1)} \leq \ldots \leq a_{(n)}, A_{(i)}$ the set $\left\{x_{(i)}, \ldots, x_{(n)}\right\}$ and where $V$ and $\mathrm{A}$ represent, respectively, the maximum and minimum.

Definition: Be $\mu$ a fuzzy measure defined on a set of criteria $X=\left\{x_{1}, \ldots, x_{n}\right\}$. The Choquet discrete integral of $n$ values $a_{1}, \ldots, a_{n}$ of $[0,1]$ is defined as follows:

$$
C_{\mu}\left(a_{1}, \ldots, a_{n}\right)=\sum_{i=1}^{n}\left(a_{(i)},-a_{(i-1)}\right) \mu\left(A_{(\mathbb{i})}\right)
$$

with the same notation as in the above definition and being also $a_{(0)}=0$.

\section{Hybrid operators}

These operators can be classified into three groups [1]:

- Operators that are constructed from the combination of a t-norm and a t-conorm. In extreme cases, these operators are t-norms or t-conorms.

- Operators, called norms, which are defined in a very similar manner to the $\mathrm{t}$-norms or $\mathrm{t}$-conorms, but with less stringent boundary conditions, and that therefore include both. This group consists of a prominent family of functions called uni-norms.

- Symmetrical adds, which are a special class of operators, and have the characteristic of being selfdual, and some, are also associative.

These operators have been widely studied; some relevant papers concerning some of them are mentioned by the way of example:

- Exponential combinations [7] [74] [75] [76].

- Interval-valued operators constructed by normal forms [77] [74].

- T-S-Aggregations [78] [79] [80] [81] [82] [83] [84] [74] [75].

- Nonlinear combinations [45] [34] [85].

- Operators constructed by additive generators [45].

- Uni-norms [86] [87].

- $\lambda$-means [8] [7].

- Symmetrical adds [88] [7] [89] [90].

\section{E. Behavioral measures}

An interesting question is to consider the attitudinal character of the aggregation operator. You can define the following measures: 
Disjunction degree (orness): the degree of orness is a measure of the tolerance of the decision-maker. Tolerant decision-makers can accept compliance with only some criteria; this corresponds to a disjunctive behavior (orness > 0.5), whose extreme example is max. On the other hand, intolerant decision-makers require that most of the criteria are equally satisfied; this corresponds to a conjunctive behavior (orness $<0.5$ ), whose extreme example is min. Of course, orness $=0.5$ corresponds to the equitable decision makers.

The concept of orness is very useful for information about the behavior of the decision-maker.

In fact, two decision-makers with the same partial evaluations $x_{1}, \ldots, x_{n}$, and same weights to the criteria, it could have even different behaviors in the sense that one of them could be tolerant and the other intolerant.

For the particular case of the OWA operators orness is [43]:

$$
\alpha(W)=\sum_{j=1}^{n} w_{j}\left(\frac{n-j}{n-1}\right) \quad \alpha \in[0,1]
$$

Dispersion: in some situations the degree of orness does not provide enough information about the true meaning of aggregation. For example, considering the median, and the arithmetic mean, which are OWA operators with weights $(0, \ldots, 1, \ldots, 0)$ and $(1 / n, \ldots, 1 / n)$ respectively, it is observed that these operators have the same degree of orness, $1 / 2$, but you can see that they are different in the sense that the first of them concentrated all the weight in a single argument. In order to capture this idea, proposed the measure of dispersion associated with the $w$ weights vector of an OWA operator:

$$
H(W)=-\sum_{j=1}^{n} w_{j} \ln \left(w_{j}\right)
$$

where $\ln$ is the neperian natural logarithm and $\ln 0=0$ by convention. This dispersion is a measure of entropy, a well-known concept already introduced in 1949 in Shannon information theory [91]. It allows us to measure the amount of information in the arguments that are used. In a sense more $\mathrm{W}$ dispersion means that it is used more information on the individual criteria in aggregation.

Balance operator: considering the OWA weights as a column vector, you can refer to the weights with low index as weights on top and those with the highest index as weights at the bottom. In this way, the weight distribution by emphasizing the value argument major/minor based on the aggregation of weights, are at the top or at the bottom of the column. In order to measure the degree of balance between the favoritism to items of greater value, or lower values, the next measure is introduced:

$$
\operatorname{Bal}(W)=\sum_{j=1}^{n} \frac{(n+1-2 j)}{n-1} w_{j} \quad \operatorname{Bal}(W) \in[-1,1]
$$

where $\operatorname{Bal}(\mathrm{W})=1$ represents an optimistic approach, $\operatorname{Bal}(\mathrm{W})=-1$ pessimistic criterion and $\mathrm{Bal}(\mathrm{W})=0$ Laplace criteria or arithmetic mean.

Divergence: finally, another interesting measure is the divergence between the weights vector. It is useful in some exceptional situations when the attitudinal character and the entropy of dispersion are not enough to analyze an aggregation weighting vector. For example, let $n=9$ the number of items aggregated and $W$ and $W^{\prime}$ the weight vectors where $w_{2}=w_{8}=0.5$ and $w_{j}=0$ for all $j \neq 2,8$; and $w_{4}^{\prime}=w_{6}^{\prime}=0.5$ and $w_{j}^{\prime}=0$ for all $j \neq 4,6$. In this case $\mathrm{H}(W)=\mathrm{H}\left(W^{\prime}\right)=\ln (2)$ and $\operatorname{Bal}(W)=\operatorname{Bal}\left(W^{\prime}\right)=0$ and cannot extract useful information from this measures. However, $\operatorname{Div}(W)=0.1406$ and $\operatorname{Div}\left(W^{\prime}\right)=0.0156$. $W^{\prime}$ vector has less divergence of $W$ vector due to the divergence between 4 and 6 is less than the difference between 2 and 8 .

$$
\operatorname{Div}(W)=\sum_{j=1}^{n} w_{j}\left(\frac{n-j}{n-1}-\alpha(w)\right)^{2}
$$

\section{CONCLUSIONS}

It was made a review about data aggregation, its main characteristics and properties. It has also been presented an overview of the main aggregation operators.

Some conditions to be taken into account to identify aggregation operators has been explained.

The classification of aggregation operators based on their behavior has been detailed and commented.

A general list of possible mathematical properties of aggregation whose verification might be desirable in certain cases has been formulated and formalized.

The main aggregation operators has been defined and explained and the main properties of them has been detailed.

The attitudinal character of the aggregation operator has been considered and the main measures of them has been defined and commented.

\section{ACKNOWLEDGMENT}

This work was performed within the framework of the accredited research project: "Decision models for synchronization of processes in distributed systems", code 12F003 - SGCyT - UNNE (General Secretary of Science and Technical - National University of the Northeast).

\section{REFERENCES}

[1] A. Pradera Gómez, Contribución al Estudio de la Agregación de Información en un Entorno Borroso, Tesis Doctoral, Universidad Politécnica de Madrid. 1999.

[2] L. Canós Darós \& V. Liern Carrión, La Agregación de Información Para la Toma de Decisiones en la Empresa, XIV Jornadas de ASEPUMA y II Encuentro Internacional, Universidad de Extremadura, España, 2006.

[3] H. Legind Larsen, Efficient importance weighted aggregation between $\min$ and max, 9th International Conference on Information Processing and Management of 
Uncertainty in Knowledge-Based Systems (IPMU'2002), Annecy, France, 2002.

[4] S. Cubillo, A. Pradera \& E. Trillas, On Joining Operators and their and / or Behaviour, Proc. International Conference IPMU'98, 673-679, Paris, France, 1998.

[5] H. J. Zimmermann, Fuzzy sets theory and its application, Kluwer Academia Publishers, Boston / Dordrecht / London, 1991.

[6] F. Herrera, E. Herrera-Viedma, J. L. Verdegay, Direct approach processes in group decision making using linguistic OWA operators, Fuzzy Sets and Systems, 79, pp. 175-190, 1996.

[7] D. Dubois \& H. Prade, A Review of Fuzzy Set Aggregation Connectives, Information Sciences 36. 85-121, 1985.

[8] G. Klir \& B. Yuan, Fuzzy Sets and Fuzzy Logic. Theory and Applications, Prentice Hall PTR, 1995.

[9] B. Bouchon-Meunier, (editor), Aggregation and Fusion of Imperfect Information, Studies in Fuzziness and Soft Computing, Vol. 12, Physica-Verlag, 1998.

[10] J. Aczél, Lectures on Functional Equations and their Applications, Academic Press, New York, USA, 1966.

[11] D. Dubois, Modèles mathématiques de l'imprécis et de l' incertain en vue d'applications aux techniques d' aide à la decision, Tesis Doctoral, Université Scientifique et Médicale de Grenoble, 1983.

[12] J. J. Dujmovié, Andness and Orsess as a Mean of Overall Importance, In: Proceedings of the IEEE World Congress on Computational Intelligence, Brisbane, Australia, June 10-15, pp. 83-88, 2012.

[13] J. J. Dujmovié \& G. De Tré, Multicriteria Methods and Logic Aggregation in Suitability Maps, International Journal of Intelligent Systems 26 (10), 971-1001, 2011.

[14] J. Fodor \& M. Roubens, On meaningfulness of means, Journal of Computational and Applied Mathematics, 103115, 1995.

[15] G. Mayor, J. Suñer \& P. Canet, Agregación multidimensional de números borrosos, Actas del VIH Congreso Español sobre Tecnologías y Lógica Fuzzy, Pamplona, España, 171-178, 1998.

[16] M. Mizumoto, Pictorial Representations of Fuzzy Connectives, Part II: cases of compensatory operators and self-dual operators, Fuzzy Sets and Systems 32, 45-79, 1989.

[17] H. T. Nguyen, V. Kreinovich \& D. Tolbert, A measure of average sensitivity for fuzzy logics, International Journal of Uncertainty, Fuzziness and Knowledge-Based Systems, Vol. 2, No. 4, 361-375, 1994.

[18] H. T. Nguyen \& E. A. Walker, A First Course in Fuzzy Logic, CRC Press, 1997.

[19] S. Ovchinnikov, Means on ordered sets, Mathematical Social Sciences 32, 39-56, 1996.

[20] S. Ovchinnikov, An Analytic Characterization of Some Aggregation Operators, International Journal of Intelligent Systems, Vol. 13, 59-68, 1998.

[21] C. Alsina, E. Trillas \& L. Valverde, Sobre conectivos lógicos no distributivos para la teoría de los conjuntos borrosos, Pub. Mat. UAB 20. 69-72, 1980.

[22] C. Alsina, E. Trillas. \& L. Valverde, On non-distributive logical connectives for fuzzy sets theory, Busefal 3, 18-29, 1983.

[23] C. Alsina, E. Trillas \& L. Valverde, On some logical connectives for fuzzy sets theory, Journal of Mathematical Analysis and Applications, Vol. 93 (1), 15-26, 1983.

[24] R. Bellman \& M. Giertz, On the analytic formalism of the theory of fuzzy sets. Inform. Sci. 5. 149-156, 1973.

[25] J. Fodor, Left-continuous t-norms in fuzzy logic: An
Overview, Acta Polytechnica Hungarica, Journal of Applied Sciences, Budapest, Hungary, 1 (2), ISSN 17858860, 2004.

[26] M. J. Frank, On the simultaneous associativity of $F(x, y)$ and $x+y-F(x, y)$, Aequationes Mathematicae 19 (2-3), 194-226, 1979.

[27] M. M. Gupta \& J. Qi, Theory of t-norms and fuzzy inference methods, Fuzzy Sets and Systems 40 (3), 431-450, 1991.

[28] C. H. Ling, Representation of Associative Functions, Publ. Math. Debrecen 12, 189-212, 1965.

[29] G. Mayor \& J. Torrens, On a class of binary operations: non-strict Archimedean aggregation functions, Proc. 18th ISMVL, Palma de Mallorca, España, 54-59, 1988.

[30] A. L. Ralescu \& D. A. Ralescu, Extensions of fuzzy aggregation, Fuzzy Sets and Systems 86, 321-330, 1997.

[31] B. Schweizer \& A. Sklar, Associative functions and statistical triangle semigroups, Publ. Math. Debrecen 8, 169-186, 1961.

[32] V. Torra, The Weighted OWA Operator, International Journal of Intelligent Systems, 12, 153-166, 1997.

[33] G. Mayor \& J. Martín, Funciones de agregación localmente internas, Actas del VIH Congreso Español sobre Tecnologías y Lógica Fuzzy, Pamplona, 355-35, 1998.

[34] R. R. Yager \& A. Rybalov, Noncommutative self-identity aggregation, Fuzzy Sets and Systems 85, 73-82, 1997.

[35] T. Calvo \& R. Mesiar, Weighted triangular norms-based aggregation operators, Fuzzy Sets and Systems, 137, pp. 310, 2003.

[36] H. Dyckhoff \& W. Pedrycz, Generalized Means as Model of Compensative Operators, Fuzzy Sets and Systems 14, 143-154, 1984.

[37] E. Trillas, S. Cubillo \& J. L. Castro, Conjunction and disjunction on ([0,1], $\leq)$, Fuzzy Sets and Systems 72, 155$165,1995$.

[38] J. M. Fernández-Salido \& S. Murakami, Extending Yager's orness concept for the OWA aggregators to other mean operators, Fuzzy Sets and Systems, 139, pp. 515-542, 2003.

[39] T. Calvo \& R. Mesiar, Aggregation operators: ordering and bounds, Fuzzy Sets and Systems, 139, pp. 685-697, 2003.

[40] J. C. Fodor, J. L. Marichal \& M. Roubens, Characterization of some aggregation functions arising from MCDM problems, en Bouchonmeunier, B.; Yager, R. R. \& Zadeh, L. A. (eds.), Fuzzy logic and soft computing, Series: Advances in fuzzy systems-Applications and theory, 4. World Scientific Singapore, pp. 194-201, 1995.

[41] R. Smolíkova \& M. P. Wachowiak, Aggregation operators for selection problems, Fuzzy Sets and Systems, 131, pp. 23-24, 2002

[42] D. Dubois \& H. Prade, Weighted minimum and maximum operations in fuzzy set theory, Information Sciences 39, 205-210, 1986.

[43] R. R. Yager, On Ordered Weighted Averaging Operators in Multicriteria Decisionmaking, IEEE Transactions on Systems, Man and Cybernetics 18, 183-190, 1988.

[44] R. R. Yager \& A. Kelman, Fusion of Fuzzy Information With Considerations for Compatibility, Partial Aggregation and Reinforcement, International Journal of Approximate Reasoning 15, 93-122, 1996.

[45] R. Mesiar \& M. Komorníková, Triangular Norm-Based Aggregation of Evidence under Fuzziness, En BouchonMeunier, B. (editor): Aggregation and Fusion of Imperfect Information, Studies in Fuzziness and Soft Computing, Vol. 12, Physica-Verlag, 11-35, 1998.

[46] D. Filev \& R. R. Yager, On the issue of obtaining OWA 
operators weights, Fuzzy Sets and Systems 94, 157-169, 1998.

[47] F. Herrera, E. Herrera-Viedma \& F. Chiclana, A study of the origin and uses of the ordered weighted geometric operator in multicriteria decision making, International Journal of Intelligent Systems 18, 689-707, 2003.

[48] X. W. Liu, Some properties of the weighted OWA operator, IEEE Transactions on Systems, Man and Cybernetics, 36, 1, 118-127, 2006.

[49] B. Llamazares, Choosing OWA operator weights in the field of Social Choice, Information Sciences: an International Journal, 177, 21, 4745-4756, 2007.

[50] J. I. Peláez \& J. M. Doña, LAMA: A Linguistic Aggregation of Majority Additive Operator, International Journal of Intelligent Systems 18, 809-820, 2003.

[51] J. I. Peláez \& J. M. Doña, Majority Additive-Ordered Weighting Averaging: A New Neat Ordered Weighting Averaging Operators Based on the Majority Process, International Journal of Intelligent Systems 18, 469-481, 2003.

[52] J. I. Peláez \& J. M. Doña, A majority model in group decision making using QMA-OWA operators, Int. J. Intell. Syst. 21, 193-208, 2006.

[53] J. I. Peláez, J. M. Doña \& A. Mesas, Majority multiplicative ordered weighting geometric operators and their use in the aggregation of multiplicative preference relations, Mathware and Soft Computing, 12, 107-120, 2005.

[54] J. I. Peláez, J. M. Doña \& A. M. Gil, Application of Majority OWA operators in Strategic Valuation of Companies, Proc Int. Eurofuse Workshop, New Trends in Preference Modelling, 2006.

[55] J. I. Peláez, J. M. Doña \& J. A. Gómez-Ruiz, Analysis of OWA Operators in Decision Making for Modelling the Majority Concept, Applied Mathematics and Computation, Vol. 186, Pages 1263-1275, 2007.

[56] J. I. Peláez, J. M. Doña \& D. L. La Red, Analysis of the Majority Process in Group Decision Making Process, JCIS 2003 (7th Joint Conference on Information Sciences), ISBN N ${ }^{\circ} 0-9707890-2-5$, Proceedings, pp. 155-159, North Carolina, USA, 2003.

[57] J. I. Peláez, J. M. Doña \& D. L. La Red, Analysis of the Linguistic Aggregation Operator LAMA in Group Decision Making Process, 32 JAIIO (Argentine Conference on Computer Science and Operational Research) - ASAI 2003 (Argentine Symposium on Artificial Intelligence), ISSN N ${ }^{\circ}$ 1666-1079, Argentina, 2003.

[58] J. I. Peláez, J. M. Doña \& D. L. La Red, Fuzzy Imputation Methods for Data Base Systems, Handbook of Research on Fuzzy Information Processing in Database, Hershey, PA, USA: Information Science Reference, 2008.

[59] J. I. Peláez, J. M. Doña, D. L. La Red \& A. Mesas, $O W A$ Aggregation with Soft Majority Operators; 33 JAIIO (33 Jornadas Argentinas de Informática e Investigación Operativa) - ASIS 2004 (Primer Simposio Argentino de Sistemas de Información), Anales 2004 (publicación electrónica en CD), ISSN N 1666-1141, Argentina, 2004.

[60] J. I. Peláez, J. M. Doña, A. Mesas \& D. L. La Red, Opinión de Mayoría en Toma de Decisiones en Grupo Mediante el Operador QMA-OWA, ESTYLF 2004 (XII Congreso Español Sobre Tecnologías y Lógica Fuzzy), Libro de Actas, págs. 449-454, ISBN N 84-609-2160-3, España, 2004.

[61] J. M. Doña, A. M. Gil, D. L. La Red \& J. I. Peláez, A System Based on the Concept of Linguistic Majority for the Companies Valuation, EconoQuantum, Vol. 8 N. 2, Guadalajara, México, 121-142, 2011.
[62] C. H. Carlsson \& R. Fuller, Fuzzy reasoning in decision making and optimization, Heidelberg: Springfield-Verlag, 2002.

[63] J. M. Merigó, M. Casanovas \& L. Martínez, L. Linguistic aggregation operators for linguistic decision making based on the Dempster-Shafer theory of evidence, International Journal of Uncertainty, Fuzziness and Knowledge-Based Systems, 18, 287-304, 2010.

[64] M. O'Hagan, Aggregating template rule antecedents in real-time expert systems with fuzzy set logic, Proc. 22nd Annual IEEE Asilomar Conf. On Signals, Systems and Computers, Pacific Grove, CA, 1988.

[65] M. Marimin, M. Umano, I. Hatono \& H. Tamura, Linguistic Labels for Expressing Fuzzy Preference Relations in Fuzzy Group Decision Making, IEEE Transactions on Systems, Man, and Cybernetics, 28(2): 205-218, 1998.

[66] L. A. Zadeh, The role of fuzzy logic in the management of uncertainty in expert systems, Fuzzy Sets and Systems, 11: 199-227, 1983.

[67] J. M. Doña Fernández, Modelado de los procesos de toma de decisión en entornos sociales mediante operadores de agregación $O W A$, Tesis doctoral, Universidad de Málaga, España, 2008.

[68] R. Yager \& D. P. Filev, Fuzzy logic controllers with flexible structures, Proc. Second Int. Cont. on Fuzzy Sets and Neural Networks, Izuka Japan, 317-320, 1992.

[69] R. R. Yager, Families of OWA operators, Fuzzy Sets and Systems, 59, 125-148, 1993.

[70] M. Grabisch, Fuzzy Integral in multicriteria decisión making, Fuzzy Sets and Systems 69, 279-298, 1995.

[71] M. Grabisch, Fuzzy measures and integrals: a survey of applications and recent issues, in Dubois, D.; Prade, H. \& Yager, R. (editors), Fuzzy Sets Methods in Information Engineering: a Guided Tour of Applications, J. Wiley \& Sons, 1996.

[72] M. Grabisch, Fuzzy Integral as a Flexible and Interpretable Tool of Aggregation, in Bouchon-Meunier, B. (editor): Aggregation and Fusion of Imperfect Information, Studies in Fuzziness and Soft Computing, Vol. 12, Physica-Verlag, 51-72, 1998.

[73] A. R. De Soto \& E. Trillas, Agregación de intervalos borrosos mediante el principio de extensión, Actas del VIII Congreso Español sobre Tecnologías y Lógica Fuzzy, Pamplona, España, 233-237, 1998.

[74] I. B. Turksen, Interval-valued fuzzy sets based on normal forms, Fuzzy Sets and Systems 20, 191-210, 1986.

[75] L. A. Zadeh, Outline of a new approach to the analysis of complex systems and decision processes, IEEE Trans. SMC, SMC-3, 38-44, 1973.

[76] H. J. Zimmermann \& P. Zysno, Latent connectives in human decision-making, Fuzzy Sets and Systems 4, 37-51, 1980.

[77] E. Trillas, A. Pradera \& S. Cubillo, A mathematical model for fuzzy connectives and its application to operator's behavioural study, in Bouchon-Meunier, B.; Yager, R. R. \& Zadeh, L. A. (eds): Information, Uncertainty, Fusion, Kluwer Scientific Publishers, 1999.

[78] C. Alsina, G. Mayor, M. S. Tomás \& J. Torrens, A characterization of a class of aggregation functions, Fuzzy Sets and Systems 53, 33-38, 1993.

[79] J. Fodor \& T. Calvo, Aggregation Functions Defined by tNorms and t-Conorms, in Bouchon-Meunier, B. (editor). Aggregation and Fusion of Imperfect Information. Studies in Fuzziness and Soft Computing, Vol. 12, Physica-Verlag, 36-48, 1998.

[80] M. K. Luhandjula, Compensatory operators in fuzzy linear 
programming with multiple objectives, Fuzzy Sets and Systems 8, 245-252, 1982.

[81] G. Mayor, Contribució a l' estudi de models matemàtics per a la lógica de la vaguetat, Tesis Doctoral, Universitat de les Illes Balears, España, 1984.

[82] G. Mayor, Sobre una classe d'operacions entre conjunts difusos: Operacions d'Agregació, Congrés Català de Lógica Matemàtica, Barcelona, España, 95-97, 1984.

[83] G. Mayor \& E. Trillas, On the representation of some aggregation functions, Proc. 16th ISMVL, Blacksburg, VA, 110-114, 1986.

[84] G. Mayor \& T. Calvo, On extended Aggregation Functions, Proc. IFSA'97, Praga, 281-285, 1997.

[85] R. R. Yager, On a class of weak triangular norm operators, Tech. Report \#MII-1528, Machine Intelligence Institute, Iona College, New Rochelle, NY, USA, 1996.

[86] J. Fodor, R. R. Yager \& A. Rybalov, Structure of Uninorms. International Journal of Uncertainty, Fuzziness and Knowledge-Based Systems, 411-427, 1997.

[87] R. R. Yager \& A. Rybalov, Uninorm aggregation operators, Fuzzy Sets and Systems 80, 111-120, 1996.

[88] J. Dombi, Basic concepts for a theory of evaluation: The aggregative operator, European J. Oper. Res. 10, 282-293, 1982.

[89] E. Klement, R. Mesiar \& E. Pap, On the relationship of associative compensatory operators to triangular norms and conorms, Int. Journal of Uncertainty, Fuzziness and Knowledge-Based Systems, Vol. 4, No. 2, 129-144, 1996.

[90] W. Silvert, Symmetric summation: A class of operations on fuzzy sets, IEEE Trans. Systems, Man Cybernet. 9, 659$667,1979$.

[91] C. E. Shannon \& W. Weaver, A mathematical theory of communication, University of Illinois Press, Urbana, 1949.

\section{Authors' Profiles}

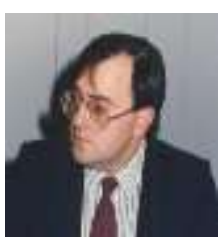

David L. la Red Martínez is currently working as Professor in Department of Computer, National University of the Northeast, Corrientes, Argentine. $\mathrm{He}$ completed her Doctorate in Systems Engineering and Computing from University of Málaga, Spain, Masters in Computer Science and Specialist University Teaching from National University of the Northeast, Corrientes, Argentine. Her areas of interests are operating systems, distributed processing, decision support systems, databases, data communications and data mining.

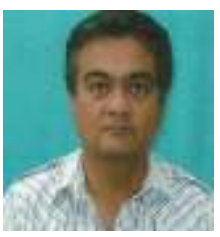

Julio C. Acosta is currently working as Professor in Department of Computer, National University of the Northeast, Corrientes, Argentine. He completed her Masters in Scientific Research Methodology from National University of Lanús, Argentine, and Specialist University Teaching from National University of the Northeast, Corrientes, Argentine. Her areas of interests are b-learning, decision support systems and fuzzy logic.

How to cite this paper: David L. La Red Martínez, Julio C. Acosta,"Aggregation Operators Review - Mathematical
Properties and Behavioral Measures", International Journal of Intelligent Systems and Applications (IJISA), vol.7, no.10, pp.63-76, 2015. DOI: 10.5815/ijisa.2015.10.08 